\title{
El paisaje lingüístico: una nueva herramienta para la enseñanza de E/LE
}

\author{
YUJING MA \\ Universitat de València \\ yujing@alumni.uv.es
}

\begin{abstract}
Resumen: El paisaje lingüístico se suele investigar dentro del campo sociolingüístico. A pesar de que han aparecido algunos estudios significativos en los que se aborda la relación relevante entre el paisaje lingüístico y la pedagogía o la didáctica, y a que algunos investigadores pioneros en el ámbito hispánico empiezan a utilizarlo como una herramienta nueva para la enseñanza de E/LE, esta orientación sigue siendo novedosa y se mantiene prácticamente inexplorada. El objetivo principal de este artículo consiste en introducir el concepto del paisaje lingüístico, realizar un repaso retrospectivo a su aplicación en la enseñanza del español, y plantear propuestas didácticas junto con un modelo detallado para demostrar que la inserción del paisaje lingüístico español puede ser una nueva dinámica para la clase de E/LE, y que los nuevos materiales de distintas modalidades consiguen fortalecer el estudio del español y ayudar a formar el aprendizaje incidental fuera del aula.
\end{abstract}

Palabras clave: paisaje lingüístico (PL), signos del paisaje lingüístico, enseñanza de E/LE, aplicación didáctica del paisaje lingüístico.

\section{The linguistic landscape: a new tool for the SFL teaching}

Abstract: The linguistic landscape is usually investigated within the sociolinguistic field. Despite the fact that there have been many significant studies with regard to the relevant relationship between the linguistic landscape and pedagogy or didactics, and some pioneers scholars in the Hispanic field have begun to use it as a new tool for the teaching of $\mathrm{E} / \mathrm{LE}$, this topic is still very novel without many explorations. Thus, the main objective of this article is to introduce the concept of the linguistic landscape, make a retrospective review of its application to the teaching of Spanish, and propose didactic proposals with a detailed model to demonstrate that the insertion of the Spanish linguistic landscape can be a new dynamic for the class of E/LE, and those new materials with different modalities can consolidate the study of Spanish and help to form incidental learning outside the classroom.

Key words: linguistic landscape (LL), signs of the linguistic landscape, teaching of SFL, didactic application of the linguistic landscape.

\section{Introducción}

Sin darnos cuenta, estamos rodeados por distintos tipos de signos lingüísticos: los rótulos comerciales de las tiendas a lo largo de las calles, los folletos de publicidad pegados sobre las paredes en cualquier rincón, las placas que nos indican las direcciones en la ciudad, todo ello pertenece al campo del paisaje lingüístico (PL). A pesar de que es un fenómeno muy común en nuestra vida diaria, el término paisaje lingüístico 
(Linguistic Landscape y la abreviatura $L L$ en inglés) fue usado por primera vez en el trabajo de Rodrigue Landry y Richard Bourhis en 1997.

En el estudio de estos autores, se investigaba a un grupo de estudiantes francófonos en varias provincias canadienses con la intención de conocer la relación entre el PL y la vitalidad etnolingüística, en este caso, la vitalidad del idioma francés y la comunidad francófona. A través del análisis, se descubría que el PL formaba una parte imprescindible en el proceso del contacto lingüístico mantenido por los individuos. De modo más significativo, los dos investigadores aportaron de partida una definición detallada del PL aceptada por todos los estudios posteriores y que se expresa así: «The language of public road signs, advertising billboards, Street names, place names, commercial shop signs, and public signs on government building combine to form the linguistic landscape of a given territory, region or urban agglomeration», (Landry / Bourhis 1997: 25).

Según Landry y Bourhis (1997), el PL dispone de dos funciones principales: la informativa y la simbólica. En primer lugar, al distinguirse de otros tipos de paisaje, el PL muestra un contenido verbal y está compuesto de uno o varios idiomas, dado que, a través del contexto lingüístico ofrecido, se puede observar la diversidad lingüística en cada zona y deducir la relación entre las distintas lenguas y su estatus social mediante el análisis de la prominencia y el predominio de cada idioma en los signos lingüísticos expuestos.

The configuration of languages present in the linguistic landscape therefore can provide important information about the diglossic nature of a particular bilingual or multilingual setting. Thus, before communicating interpersonally with a single inhabitant, one can use the linguistic landscape as an indicator of the power and status relationship that exists between the various language groups present within a given administrative or geographical region.

Landry / Bourhis (1997: 26)

Además, el PL se encarga de mostrar y difundir informaciones de distintos ámbitos de la sociedad con distintas finalidades hacia los receptores; por ejemplo, la publicidad o los carteles privados intentan vender mercancías o servicios al público, mientras que los signos surgidos en el ámbito administrativo del gobierno transmiten información necesaria a sus ciudadanos o tratan de influir en ellos bajo las orientaciones de la política lingüística, tales como los nombres de los edificios gubernamentales o los de las calles.

En segundo lugar, aparte del propio contenido que se transmite a través del PL, la forma en la que se expresan estas informaciones también constituye una función considerable dentro del PL. Las diversas lenguas que se encuentran en el PL representan a los diferentes grupos lingüísticos que hay tras ellos, y afectan directamente a los hablantes de dichos idiomas. En otras palabras, los signos lingüísticos presentan un efecto afectivo para los grupos lingüísticos y muestran la vitalidad tanto de los idiomas como de las propias comunidades lingüísticas en su totalidad. Como señala Bourhis (1992 apud Landry / Bourhis 1997: 27): «The absence or presence of one's own language on public signs has an effect on how one feels as a member of a language group within a bilingual or multilingual setting».

Landry / Bourhis (1997) dividen los signos lingüísticos en dos grupos generales al 
considerar los distintos grupos de emisores: signos privados y signos públicos. Los signos privados se refieren a los que producen individuos, asociaciones o empresas privadas que funcionan autónomamente $\mathrm{y}$, por el contrario, los signos públicos, también denominados signos institucionales, están emitidos por las autoridades, tales como gobiernos nacionales o municipales, grupos o asociaciones públicas. Siguiendo este tipo de clasificación sobre los signos lingüísticos dentro del PL, Ben-Rafael et al. (2006: 10) introducen la concepción de signos top-down y bottom-up. Los signos top-down ('arriba-abajo') se refieren a los signos públicos, y los signos bottom-up ('abajo-arriba') son los privados. Según estos autores, la diferencia principal entre estas dos categorías consiste en que los primeros son signos en los que se espera por parte de los receptores que reflejen un compromiso general hacia la cultura dominante, mientras que los segundos están diseñados libremente según las distintas estrategias individuales.

Aunque el estudio del PL es todavía novedoso en comparación con otros ámbitos lingüísticos, las investigaciones también se han extendido en el campo sociolingüístico y se ha aplicado a distintos ámbitos de las ciencias sociales. En este sentido, algunos estudios tales como la investigación de Comajoan (2013) sobre la situación lingüística en Cataluña, el estudio de Backhaus (2009) para asegurar la vitalidad del idioma francés y las políticas lingüísticas en Quebec, o el análisis de Wu / Techasan (2016) acerca del uso del tailandés y el chino estipulado por el gobierno nacional, manifiestan que los idiomas aparecidos dentro del PL, especialmente en los signos públicos, muestran parcialmente la política lingüística local, y que en varias situaciones, el PL y las políticas lingüísticas aplicadas en un territorio se influyen recíprocamente.

Además, también son fundamentales otros trabajos que se encargan de demostrar el multilingüismo urbano revelado por el PL, o el contacto de idiomas en un PL bilingüe o multilingüe. Entre ellos se destacan el estudio de Muñoz Carrobles (2010) y Sáez Rivera / Castillo Lluch (2012) que abordan el PL en Madrid desde la perspectiva del multilingüismo, y la investigación de Esteba Ramos (2014) sobre el contacto del español con el inglés u otros idiomas extranjeros y la interferencia que recibe la variedad andaluza en el PL de Málaga.

\section{El paisaje lingüístico y la enseñanza}

Al constituir un fenómeno diario y accesible para aprendices de lenguas de todos los perfiles, el PL puede ofrecer abundantes materiales nuevos para el campo pedagógico y didáctico, y muy especialmente para la enseñanza de idiomas. La utilización del PL como una herramienta para despertar la conciencia sobre las lenguas y la diversidad cultural, para desarrollar una competencia multilingüe y para promover la comprensión y tolerancia hacia diferentes grupos étnicos ha llamado recientemente la atención de los investigadores.

\subsection{Relevancia de la aplicación del PL en la enseñanza}

Algunos de los investigadores pioneros que han indagado acerca de la función pedagógica y didáctica del PL, como Cenoz / Gorter (2008), han señalado especialmente su contribución a la adquisición de una segunda lengua. En primer lugar, el PL sirve como un input para el aprendizaje. Considerando que el PL tiene una accesibilidad extremadamente fácil para los hablantes, influye sobre la gente 
diariamente y ayuda al aprendizaje incidental, de modo que en este sentido se ha relacionado con las investigaciones sobre el aprendizaje de lenguas en el extranjero que han mostrado la influencia positiva del contacto directo con la lengua objeto. En cuanto al input ofrecido por el PL, Cenoz / Gorter (2008) han declarado sus ventajas, dado que es auténtico y se presenta contextualizado al ser una parte de la trama social. En segundo lugar, las diversas funciones y actores que se encuentran detrás de los signos lingüísticos del paisaje ayudan a desarrollar una competencia pragmática. En tercer lugar, otra de las ventajas se halla en que el PL puede realizar la combinación de la comprensión semiótica y la comprensión de los textos escritos, de modo que ayuda a mejorar las habilidades de la alfabetización. Por último, el PL sirve para el perfeccionamiento de las competencias multilingües, ya que las informaciones ofrecidas por los signos multilingües no son necesariamente iguales, de modo que lo más importante es entender cada idioma considerando su propia cultura y sus características lingüísticas para deducir las intenciones subyacentes.

\subsection{Estudios antecedentes sobre la enseñanza del español a través del PL}

Considerando que el PL es un campo novedoso, existe una escasez en cuanto a su aplicación a la pedagogía lingüística $\mathrm{y}$, especialmente, a la enseñanza de segundas lenguas. Dentro de las aportaciones realizadas hasta momento, existe una cantidad notable de investigadores que han utilizado el PL como un escenario de materiales nuevos para la enseñanza del inglés, mientras que en el ámbito hispánico la aplicación del PL a la enseñanza no ha recibido mucha atención por parte de los profesores y sigue siendo un tema relevante por explorar.

Esteba Ramos (2013), a través de su estudio piloto exploratorio sobre la aplicación del PL en el aula de E/LE, ha declarado que el PL permite a los alumnos a mantener el contacto con distintos tipos textuales y ofrece muestras reales del uso práctico del español. En cuanto a las propuestas didácticas planteadas en su artículo, la autora señala que disponen de dos ventajas principales. En primer lugar, las muestras -en este caso se refiere a los signos lingüísticos dentro del PL-, se pueden encontrar fácilmente y de forma gratuita. En segundo lugar, el desarrollo de las nuevas tecnologías, tales como internet y las redes sociales, hace que las propuestas se realicen con más posibilidades de éxito y eficiencia.

Según sus propuestas dirigidas hacia diferentes niveles ofrecidos por el $\mathrm{MCER}^{1}$, a los alumnos de los niveles A1 y A2 les puede pedir que recopilen fotografías de los signos lingüísticos con la aparición del español en su propia ciudad para «concienciar al alumno de que sabe más palabras en español de las que pueda imaginar» (Esteba Ramos 2013: 476). Desde las fotografías recogidas, el profesor elige elementos adecuados según el nivel de conocimiento de sus alumnos. A partir del nivel A2, considerando que los alumnos ya tienen una base léxica suficiente, se puede ampliar el ámbito de búsqueda e incorporar la utilización de las $\mathrm{TIC}^{2}$. En este nivel de enseñanza, la autora propone una actividad en la que el profesor puede elegir una cantidad de ciudades hispanohablantes que están disponibles a través de Street View en la página web de

\footnotetext{
${ }^{1}$ Marco Común Europeo de Referencias para las lenguas. Según este marco, hay seis niveles para medir las capacidades de los aprendizajes de determinados idiomas: A1, A2 (nivel básico), B1, B2 (nivel intermedio) y C1 y C2 (nivel avanzado).

2 TIC: Tecnología de la Información y Comunicación.
} 
Google Maps, y pedir a los alumnos la selección de signos lingüísticos que les gusten durante un mes. Basándose en estos materiales, al finalizar el periodo de preparación se realizan presentaciones en la clase para describirlos. Además, y como aportación participativa de los resultados, estas presentaciones se suben a la red social para que otros aprendices del español también tengan acceso a las informaciones e interactúen con los estudiantes de esta clase.

Para los niveles más avanzados, a partir del B2, las propuestas no se focalizan simplemente en la discusión entre los alumnos sobre la información ofrecida en el PL, sino que se profundiza en la búsqueda de errores lingüísticos dentro de los signos y se propone su corrección. Al mismo tiempo, a partir de estos materiales, los profesores pueden orientar a los aprendices a analizar los signos según diferentes niveles de error, tales como errores ortográficos, sintácticos o morfológicos.

No solo Esteba Ramos ha diseñado actividades relacionadas con los materiales que pueden proporcionar los signos del PL en los niveles de aprendizaje inicial y medio. Aladjem y Jou (2016), de la Universidad de Tel Aviv, también han diseñado una actividad similar a la anterior al aplicar el PL para un curso universitario del español, con el objetivo de despertar la conciencia de los alumnos sobre el aprendizaje incidental dentro del ambiente diario. En esta actividad, los estudiantes también tienen que recopilar signos del PL relacionados con el español en su vida diaria. Los datos recogidos pueden ser de varias modalidades, incluyendo fotografías, vídeos, audios, etc. Para analizar estos materiales, los profesores piden a los alumnos que los suban a una cuenta de Facebook específica para esta actividad junto con descripciones cortas en español, a través de las cuales los alumnos pueden expresar sus propias observaciones o reflexiones sobre los signos lingüísticos. Durante el proceso del análisis, los profesores ayudan a los alumnos a analizar tanto el aspecto visual como el textual de los materiales obtenidos del PL.

Además de los estudios mencionados, otros investigadores, aparte de utilizar el PL en la enseñanza, lo ha empleado junto con otras teorías didácticas; por ejemplo, Acevedo Aguilar (2015) ha utilizado los signos lingüísticos del PL con distintos enfoques en el aula de E/LE para mejorar diferentes destrezas y Soto Rubio (2017) ha combinado la teoría del PL con la de las inteligencias múltiples (IIMM) ${ }^{3}$.

A través de las propuestas didácticas mencionadas se constata que el PL nos puede servir como otra herramienta nueva para completar y mejorar las maneras tradicionales en la enseñanza del español, y que sobre todo enriquece los materiales lingüísticos más allá de los que se encuentran en los manuales con muestras lingüísticas reales y auténticas obtenidas por los propios aprendices. Teniendo en cuenta las ventajas sobre la aplicación del PL y aprovechando las tecnologías actuales, en 2013 Coronada creó un blog cuyo nombre es "Aprende español callejeando por Madrid". Este blog se dedica a mostrar los signos lingüísticos recogidos por las calles en Madrid con el fin de transmitir un conocimiento tanto lingüístico como cultural del español. Se suele colgar varias fotografías que contienen signos sobre un tema parecido con una breve redacción como explicación debajo de la fotografía (Imagen 1). Este blog, por un lado, muestra el

\footnotetext{
${ }^{3}$ En este artículo, se ha adoptado el concepto de IIMM planeado por Gardner (1983). Según él, existe en total ocho tipos de inteligencias: inteligencia lógico-matemática, inteligencia lingüístico-verbal, inteligencia cinésico-corporal, inteligencia visual-espacial, inteligencia naturalista, inteligencia interpersonal e inteligencia intrapersonal (Soto Rubio 2017: 10).
} 
PL de Madrid y puede ser útil para los investigadores del PL y, por el otro, ayuda significativamente a los aprendices del español, especialmente a los aprendices extranjeros que han vuelto a su país, para que mantengan el contacto con el español, lo cual también fue el motivo inicial para la creación este blog.

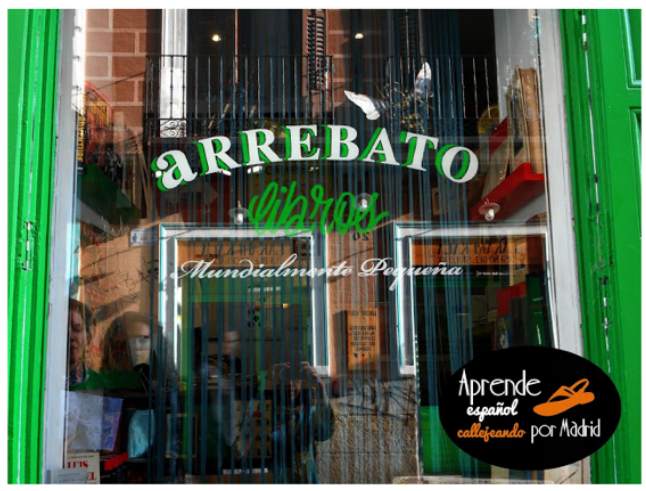

emocion; digo ataque porque es la tlegada de un sentir We hablamos, por ejemplo, de un arrebato de alegria o de un arrebato de ira. Esos arrebatos nos impulsan a acciones inesperadas, repentinas. Y los libros nos pueden causar también emociones intensas, arrebatos de emociones.

Imagen 1: Análisis de un signo lingüístico en el blog

\section{Diseño de actividades relacionadas con el PL en el aula de español}

Tras el repaso a la teoría del PL y a los trabajos previos más significativos de su aplicación a la enseñanza de lenguas, se observa aún más claramente que el PL puede ofrecer materiales esencialmente auténticos sobre el uso práctico de un idioma, por lo que el conocimiento adquirido a partir de dichos materiales se ajusta al desarrollo lingüístico y social. Los materiales lingüísticos extraídos desde el PL complementan a los materiales tradicionales ofrecidos por los manuales con fenómenos novedosos del idioma y también sustituyen o pueden actualizar los contenidos anticuados.

Además, los signos lingüísticos que aparecen en el PL permiten a los estudiantes aprender el idioma a través de diferentes modalidades y contenidos, tales como menús de los restaurantes, avisos públicos, carteles de horarios o de advertencias, etc. La capacidad de interpretar estos signos no solo ayuda a mejorar la competencia lingüística, sino que también favorece la preparación del examen de DELE, porque en las pruebas de los niveles iniciales (A1-A2) la orientada hacia la compresión de lectura suele incidir en ellos y se suele encontrar una cantidad considerable de ejercicios diseñados a partir de los signos que se observan en la vida diaria (imágenes 2 y 3 ).
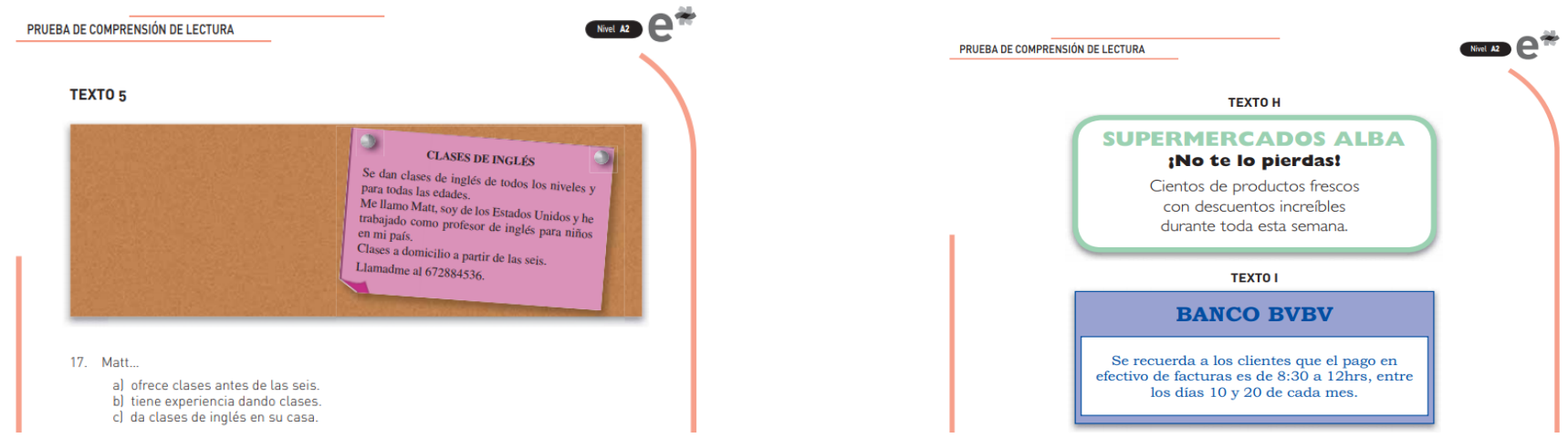


\subsection{Preparación previa para la aplicación del PL al aula de $E / L E$}

En cuanto a la aplicación del PL en el aula de idiomas, en este caso al aprendizaje del español como lengua extranjera, la clave se sitúa en cómo diseñar unas actividades que pueden combinar adecuadamente los signos lingüísticos del PL con el conocimiento del español de los aprendices. En la propuesta que vamos a realizar a continuación es fundamental tener en cuenta una serie de cuestiones previas que modelarán en la ficha que se presenta más abajo (tabla 1) que concreta los pasos previos para la preparación de una clase de E/LE a partir del PL y que pasamos a exponer.

Generalmente, según las propuestas mencionadas, hay dos participantes importantes en la aplicación del PL a la enseñanza con diferentes funciones en el proceso: en primer lugar, el profesor, el cual desempeña un papel imprescindible tanto en el diseño de las actividades como en la orientación académica en el aula $\mathrm{y}$, en segundo lugar, los aprendices, quienes, como receptores de los conocimientos, no pueden ocupar un puesto pasivo, sino que deben desarrollar necesariamente una actitud activa. Así que a través de las actividades diseñadas se pretende que los alumnos se vean obligados a reflexionar sobre los signos del PL no solo desde la perspectiva gramatical, sino también desde sus contenidos pragmáticos, culturales, sociales, etc.

Teniendo en cuenta el papel del profesor como un guía y con el fin de organizar eficazmente una clase de E/LE a través del aprovechamiento del PL se procede a la preparación de la clase según los pasos que muestra la tabla 1. Previamente, hay que considerar la fecha y la duración de cada clase con antelación para ajustarse a la enseñanza diaria y completar el contenido y las actividades tradicionales en el aula puesto que este nuevo modelo de enseñanza no está pensado para sustituir a las clases regulares, sino que sirve como complemento o dinámica para enriquecer las formas didácticas y facilitar a los alumnos el idioma de facto.

Los materiales se deben seleccionar adecuadamente según el nivel aplicable para que los alumnos puedan entenderlos con su conocimiento actual de lengua. La forma de agrupación depende de las actividades concretas y varía según la necesidad de cada sesión de la clase. El objetivo general para cada clase del PL presenta dos partes. Por un lado, se dedica a aprender algún aspecto de gramática del español, punto sustancial para una clase de E/LE, y por el otro, la enseñanza puede extenderse hacia el aspecto cultural o social a través de los contenidos de los signos, tanto lingüísticos como icónicos, de manera que los alumnos pueden avanzar en su aprendizaje del español mientras mejoran su capacidad lingüística contextualizada. Después, en función de las diferentes destrezas que necesiten mejorar los alumnos y los objetivos detallados preparados por los profesores sobre los signos analizados, se organizan las sesiones con actividades durante la clase no solo deben estar concentradas en el contenido lingüístico de los signos, puesto que siempre se puede añadir informaciones relacionadas para que la enseñanza y el análisis sean más sintéticos. 
Preparación de una clase con materiales del PL

Fecha de la clase

Nivel aplicable

Materiales preparados (Fotografias de los signos lingüísticos)

Duración

Agrupación: Individual o en grupos

Destrezas para desarrollar

Contenido lingüístico (análisis gramatical)

Comentarios sobre los signos

Objetivos detallados

Tabla 1: Preparación para una clase de E/LE con materiales del PL

\subsection{Propuesta de un modelo didáctico}

A continuación, mostraremos un modelo detallado de nuestra propuesta para la organización de una clase de E/LE con la ayuda del PL y sus aplicaciones. En este modelo didáctico, a partir de los signos del PL (Imágenes 4 y 5), se especifican los objetivos y la temporización para ofrecer una referencia a los futuros investigadores que tengan interés en este tema (Tabla 2).

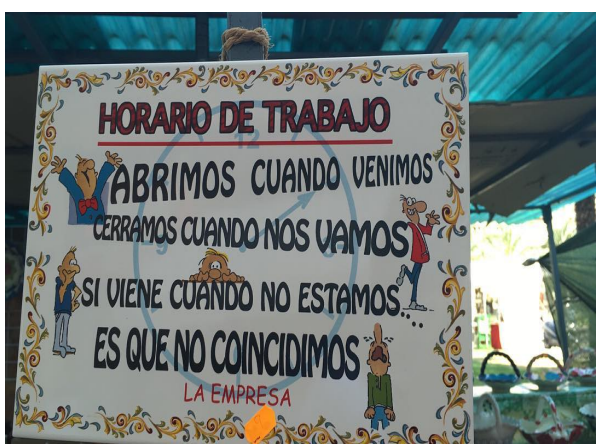

Imagen 4

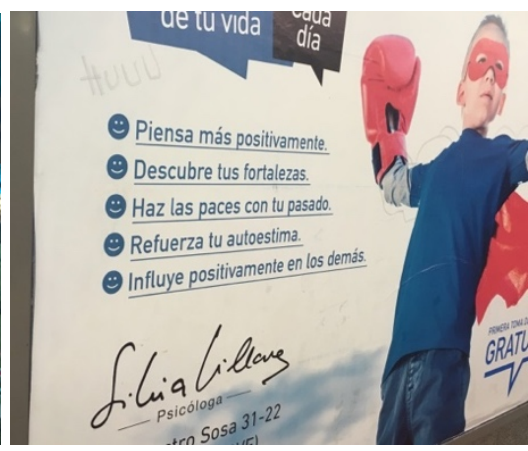

Imagen 5 


\begin{tabular}{|c|c|c|}
\hline \multicolumn{3}{|c|}{ Modelo de una clase de E/LE con materiales del PL } \\
\hline Fecha de la clase: & & $20 / 09 / 2018$ \\
\hline Nivel aplicable: & & $\mathrm{B} 1-\mathrm{B} 2^{4}$ \\
\hline Duración: & & 1 hora \\
\hline Materiales preparados: & \multicolumn{2}{|r|}{2 Fotografias (Imágenes 4 y 5 ) } \\
\hline Agrupación: & & Individual + En grupos \\
\hline Destrezas: & \multicolumn{2}{|c|}{ Destreza oral / Destreza comprensiva } \\
\hline \multirow[t]{2}{*}{$\begin{array}{l}\text { Contenido lingüístico ( } 45 \mathrm{mins}) \text { : } \\
\text { En esta sesión, se enseña la gramática y } \\
\text { se diseñan ejercicios para que los alumnos los } \\
\text { hagan de forma individual con el objetivo de } \\
\text { fortalecer el aprendizaje. }\end{array}$} & Imagen 1 & $\begin{array}{l}\text { - Uso del relativo "cuando" con } \\
\text { indicativo / subjuntivo (B1) } \\
\text { - Oraciones subordinadas } \\
\text { adverbiales que expresan la } \\
\text { simultaneidad introducidas por } \\
\text { "cuando" (B1-B2) } \\
\text { - Uso y significado de la palabra } \\
\text { "coincidir" y sus sinónimos } \\
\text { (B2) } \\
\text { - Uso coloquial de "es que..." } \\
\text { para dar una explicación con } \\
\text { forma atenuada }\end{array}$ \\
\hline & Imagen 2 & $\begin{array}{l}\text { - Uso del imperativo con valor de } \\
\text { sugerencia o consejo (B1) } \\
\text { - Uso y significado de las palabras } \\
\text { "fortaleza / reforzar/ autoestima / } \\
\text { psicóloga" } \\
\text { - Uso de la combinación fija } \\
\text { "influir en..." (B2) }\end{array}$ \\
\hline
\end{tabular}

${ }^{4}$ La selección del contenido lingüístico para distintos niveles se puede decidir al consultar el Plan Curricular del Instituto Cervantes, pero siempre se puede profundizar y simplificar el contenido gramatical según la necesidad de la enseñanza y el tiempo disponible. 


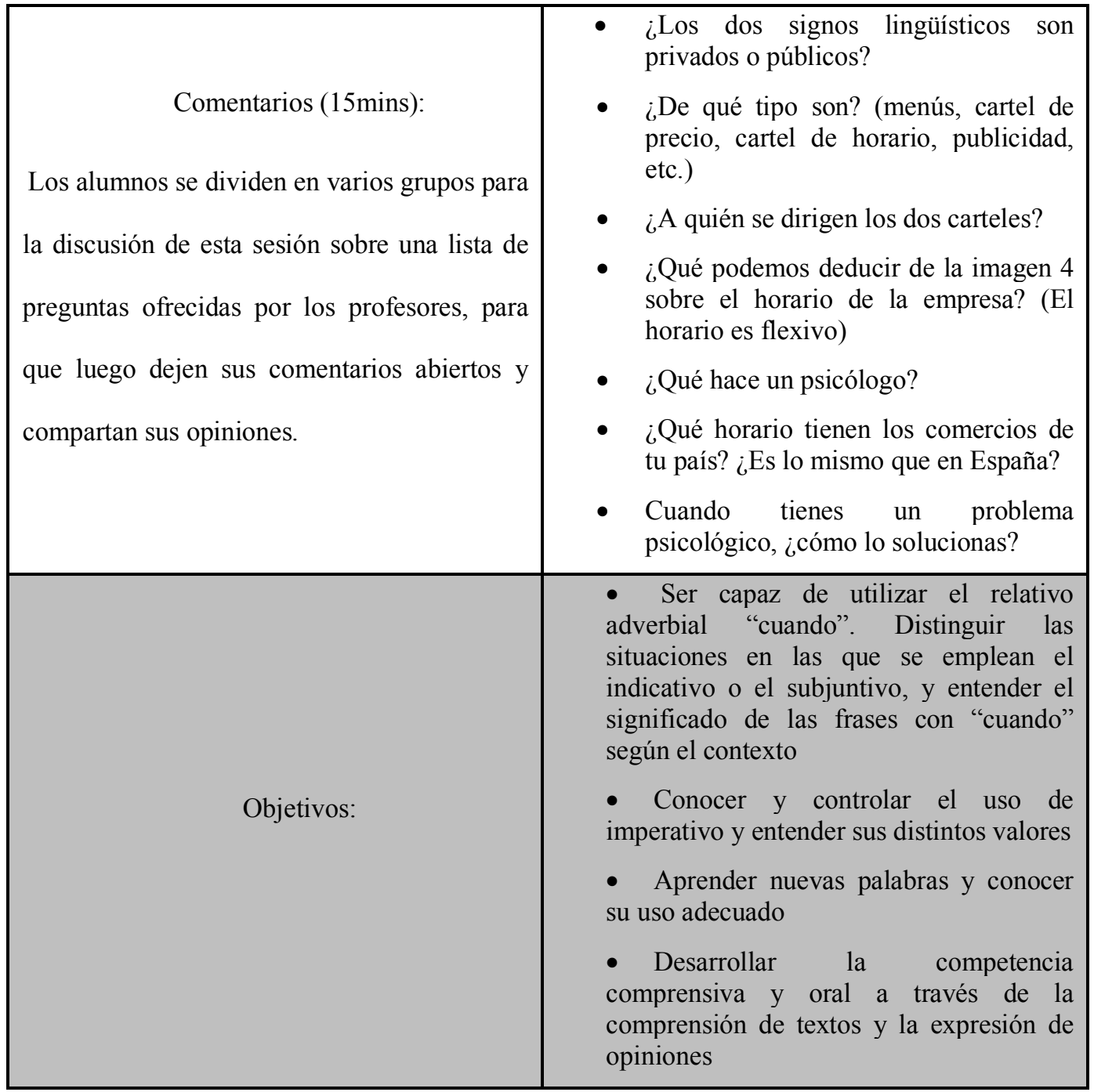

Tabla 2: Modelo de la enseñanza con el PL

\section{Conclusión}

Como se ha mostrado a lo largo de esta exposición, aunque el PL sea una aproximación a los hechos lingüísticos y sociales bastante novedosa, lo es particularmente en el ámbito de la enseñanza de segundas lenguas, $y$, en especial, del español. Y así mismo es innegable que el PL ocupa una parte importante en nuestra vida diaria, y que dispone de la ventaja de ofrecer de modo fácil y accesible materiales lingüísticos auténticos. Son varias sus ventajas: lo que reflejan los materiales del PL es el uso práctico del español y en textos con modalidades muy variadas $\mathrm{y}$, por tanto, es muy relevante su aprovechamiento y aplicación a la enseñanza de E/LE para desarrollar distintas destrezas lingüísticas. De modo secundario, pero también importante, la información de los signos del PL y la coincidencia con los materiales que eventualmente se emplean en el DELE, permite a los estudiantes familiarizarse con las muestras de lengua real empleada en las pruebas. Por último, y lo que consideramos fundamental, las actividades vinculadas a los signos lingüísticos del PL con la aparición del español ayudan a despertar la conciencia lingüística de los alumnos y a integrar el aprendizaje incidental y, al mismo tiempo, hacen que la enseñanza y el aprendizaje se extiendan por 
cualquier rincón de la ciudad y que los alumnos se den cuenta de que su entorno vital también es un aula del español.

\section{Bibliografía}

Acevedo Aguilar, Clara (2015). El paisaje lingüistico en la enseñanza de ELE: primera aproximación. Recuperado el 18/09/18, de: https://rua.ua.es/dspace/bitstream/10045/75027/1/TFM_Clara-Acevedo-Aguilar.pdf

AlADJEM, Ruthi y JoU, Bibiana (2016). «The linguistic landscape as a learning space for contextual language learning». Journal of Learning Space, 5(2), 66-70.

BACKHAUS, Peter (2009). «Rules and regulations in linguistic landscape: a comparative perspective». En Elena Shohamy y Durk Gorter (ed.); Linguistic Landscape: Expanding the Scenery (pp. 157-172). Nueva York: Routledge.

CENOZ, Jasone y GORTER, Durk (2008). «The linguistic landscape as an additional source of input in second language acquisition». International Review of Applied Linguistics in Language Teaching, 46(3), 267-287.

COMAJOAN COLOMÉ, Llorenç (2013). «El paisaje lingüístico en Cataluña: caracterización y percepciones del paisaje visual y auditivo en una avenida comercial de Barcelona». Revista internacional de lingüistica hispánica, 21, 63-88.

Esteba Ramos, Diana (2013). «¿Qué te dice esta ciudad? Modelos de reflexión y propuestas de actuación en torno al paisaje lingüístico en la clase de Español Lengua Extranjera». En Leonel Ruiz Miyares et alii (ed.); Actualizaciones en Comunicación Social (pp. 474-479). Santiago de Cuba: Centro de Lingüística Aplicada.

EsteBA RAMOS, Diana (2014). «Aproximación del paisaje lingüístico de Málaga: préstamos y reflejos de una realidad lingüística plural». Recherches, 12, 165-187.

MA, Yujing (2017). «El paisaje lingüístico de la ciudad de Valencia: una aproximación a su estudio». Lengua y Migración, 9 (1), 63-84.

MuÑoz Carrobles, Diego (2010). «Breve itinerario por el paisaje lingüístico de Madrid». Ángulo Recto, 2(2), 103-109.

Wu, Hongmei y TeCHASAN, Sethawut (2016). «Chinatown in Bangkok: the multilingual landscape». MANUSYA: Journal of Humanities. 22, 38-52.

SÁEz RiverA, Daniel Moisés y CAStillo Lluch, Mónica (2012). «The Human and Linguistic Landscape of Madrid (Spain)». En Christine Hélot, Monica Barni, Carla Bagna y Rudi Janssens (ed.); Linguistic Landscapes, Multilingualism and Social Change (pp. 309-328). Bern: Peter Lang.

Soto-Rubio, María Gabriela (2017). Paisaje lingüístico e inteligencias múltiples en la enseñanza de español como lengua extranjera. Trabajo fin de máster en Estudios Hispánicos Avanzados. Valencia: Universitat de València. 